\title{
Complete treatment of double surface weakly singular integrals arising in Galerkin mixed potential integral equation formulations via the direct evaluation method
}

\author{
Athanasios G. Polimeridis* and Juan R. Mosig \\ Laboratory of Electromagnetics and Acoustics (LEMA) \\ Ecole Polytechnique Fédérale de Lausanne (EPFL) \\ CH-1015 Lausanne, Switzerland \\ E-mail: athanasios.polymeridis@epfl.ch; juan.mosig@epfl.ch
}

\section{Introduction}

Mixed potential integral equation (MPIE) based algorithms stand as one of the most accurate and efficient tools in computational electromagnetics, especially when dealing with electromagnetic scattering and radiation problems from arbitrary perfect conducting surfaces [1], [2]. The numerical solution of the surface MPIE via the method of moments, using the linear Rao-Wilton-Glisson basis functions [1], calls for the computation of the 4-D weakly singular integrals:

$$
I_{p, q}^{\text {sing }}=\int_{E_{P}} \zeta_{p} \int_{E_{Q}} g\left(\mathbf{r}, \mathbf{r}^{\prime}\right) \zeta_{q}^{\prime} d A_{Q} d A_{P}, \quad p, q=1,2,3
$$

and

$$
I_{s}^{\text {sing }}=\int_{E_{P}} \int_{E_{Q}} g\left(\mathbf{r}, \mathbf{r}^{\prime}\right) d A_{Q} d A_{P}
$$

where the two triangular elements $E_{P}$ and $E_{Q}$ may coincide (coincident integration), share a common edge (edge adjacent integration), or share a common vertex (vertex adjacent integration). $A_{P}, A_{Q}$ are the associated triangular surfaces, $g\left(\mathbf{r}, \mathbf{r}^{\prime}\right)=e^{-j k R} / R$ is the free-space Green's function, $R=\left|\mathbf{r}-\mathbf{r}^{\prime}\right|$ is the distance function, $k$ is the wavenumber of the medium and $\zeta_{p}, \zeta_{q}^{\prime}$ are simplex coordinates associated to arbitrary vertices of the triangular elements.

Here, a further extension of [3] for the edge adjacent and the vertex adjacent cases is presented, thus, completing the semi-analytical treatment of weakly singular integrals on planar triangles via the direct evaluation method [4].

\section{Direct Evaluation Method}

The first step of the direct evaluation method is to introduce the equilateral parameter space $\{\eta, \xi\}$, where $-1 \leq \eta \leq 1,0 \leq \xi(\eta) \leq \sqrt{3}(1-|\eta|)$. The weakly singular integrals in (1), (2) can be evaluated as a linear combination of the following terms:

$$
I_{p, q}=\int_{-1}^{1} d \eta \int_{0}^{\xi(\eta)} \psi_{p}(\eta, \xi) d \xi \int_{-1}^{1} d \eta^{\prime} \int_{0}^{\xi\left(\eta^{\prime}\right)} \psi_{q}\left(\eta^{\prime}, \xi^{\prime}\right) \frac{e^{-j k R}}{R} d \xi^{\prime}
$$

$\psi_{1}(\eta, \xi)=1, \psi_{2}(\eta, \xi)=\eta$ and $\psi_{3}(\eta, \xi)=\xi$. 


\section{Edge Adjacent Case}

In the edge adjacent integration, we employ two polar coordinate transformation to cancel the line of singularity defined by $\xi=\xi^{\prime}=0$ and $\eta=-\eta^{\prime}$ :

$$
\eta^{\prime}=\rho \cos (\theta)-\eta, \quad \xi^{\prime}=\rho \sin (\theta) \quad \text { and } \quad \rho=\Lambda \cos (\Psi), \quad \xi=\Lambda \sin (\Psi) .
$$

The distance function in the new parametric system takes the form $R=\Lambda B(\theta, \Psi)$ and the original integral can be written as a sum of 3-D integrals,

$$
I_{p, q}=\sum_{l=0}^{1} \sum_{m=0}^{1} \int_{-1}^{1} d \eta \int_{\Theta_{l}}^{\Theta_{l+1}} d \theta \int_{\Psi_{m}}^{\Psi_{m+1}} A_{p, q}(\eta, \theta, \Psi) d \Psi
$$

since the integral with respect to $\rho$,

$$
A_{p, q}(\eta, \theta, \Psi)=\frac{\cos (\Psi)}{B(\theta, \Psi)} \int_{0}^{L(\eta, \theta)} \psi_{p}(\eta, \Psi, \Lambda) \psi_{q}(\eta, \theta, \Psi, \Lambda) e^{-j k B(\theta, \Psi) \Lambda} \Lambda d \Lambda
$$

can be evaluated analytically.

Moving to the second analytical integration, it is convenient to consider $\eta$ positive and negative separately. In the following, we examine the integration over region $\eta \geq$ 0 . Moreover, in the implementation of this method, integration over positive values of $\eta$ suffices, since integration over region $\eta \leq 0$ can be easily computed by flipping the elements around and using the $\eta \geq 0$ formulas. The additional complication in the edge adjacent case is that both the $\theta$ and $\Psi$ integrals are separated in two pieces. In particular, the splitting in $\theta$ is $0<\theta \leq \Theta_{1}$ and $\Theta_{1} \leq \theta \leq \pi$. Finally, after re-ordering the integral and integrating with respect to $\eta$ analytically for both cases, the weakly singular integrals for $\eta>0$ have been reduced to the following 2-D smooth integrals:

$$
\begin{aligned}
I_{p, q}^{\eta_{+}} & =\int_{\pi / 2}^{\pi} d \theta \int_{0}^{\Psi_{1}^{+}} X_{p, q}^{a} d \Psi+\int_{\pi / 3}^{\pi / 2} d \theta \int_{0}^{\Psi_{1}^{+}} X_{p, q}^{b} d \Psi+\int_{\pi / 2}^{\pi} d \theta \int_{\Psi_{1}^{+}}^{\pi / 2} X_{p, q}^{c} d \Psi \\
& +\int_{\pi / 3}^{\pi / 2} d \theta \int_{\Psi_{1}^{+}}^{\pi / 2} X_{p, q}^{d} d \Psi+\int_{0}^{\pi / 3} d \theta \int_{0}^{\Psi_{00}} X_{p, q}^{e} d \Psi+\int_{\pi / 3}^{\pi / 2} d \theta \int_{0}^{\Psi_{\theta}} X_{p, q}^{f} d \Psi \\
& +\int_{\pi / 3}^{\pi / 2} d \theta \int_{\Psi_{\theta}}^{\Psi_{00}} X_{p, q}^{g} d \Psi+\int_{0}^{\pi / 3} d \theta \int_{0}^{\Psi_{00}} X_{p, q}^{h} d \Psi+\int_{0}^{\pi / 3} d \theta \int_{\Psi_{00}}^{\pi / 2} X_{p, q}^{i} d \Psi \\
& +\int_{\pi / 3}^{\pi / 2} d \theta \int_{\Psi_{\theta}}^{\Psi_{00}} X_{p, q}^{k} d \Psi+\int_{\pi / 3}^{\pi / 2} d \theta \int_{\Psi_{00}}^{\pi / 2} X_{p, q}^{l} d \Psi
\end{aligned}
$$

where the integrands are well-behaved functions of $(\theta, \Psi)$. It is worth mentioning in this point that the dimensionality reduction of the original 4-D integrals in both the edge adjacent case and vertex adjacent case (as will be shown next) is limited compared to the coincident case due to the algebraic complexity of the distance function after the variable transformations. To be more precise, the dimensionality of the final integrals is equal to the independent variables in the expressions $\alpha(\theta)$ [3], $B(\theta, \Psi)$ and $\Gamma\left(\theta_{p}, \theta_{q}, \Psi\right)$ for, respectively, the coincident, edge adjacent and vertex adjacent case. 
Similarly to the coincident integration [3], the remaining case (integration over negative values of $\eta$ ) can be handled by simply flipping the master triangles (triangles in the equilateral triangle parametric space) and employing the formulas for the $\eta>0$

case. Finally, the weakly singular integrals $I_{p, q}$ can be evaluated as a function of the subintegrals $I_{p, q}^{\eta_{+}}$and $I_{p, q}^{\eta_{-}}$,

$$
\begin{array}{llll}
I_{1,1}=I_{1,1}^{\eta_{+}}+I_{1,1}^{\eta_{-}}, & I_{1,2}=I_{1,2}^{\eta_{+}}-I_{1,2}^{\eta_{-}}, & I_{1,3}=I_{1,3}^{\eta_{+}}+I_{1,3}^{\eta_{-}}, & I_{2,1}=I_{2,1}^{\eta_{+}}-I_{2,1}^{\eta_{-}}, \\
I_{2,2}=I_{2,2}^{\eta_{+}}+I_{2,2}^{\eta_{-}}, & I_{2,3}=I_{2,3}^{\eta_{+}}-I_{2,3}^{\eta_{-}}, & I_{3,1}=I_{3,1}^{\eta_{+}}+I_{3,1}^{\eta_{-}}, & I_{3,2}=I_{3,2}^{\eta_{+}}-I_{3,2}^{\eta_{-}}, \\
I_{3,3}=I_{3,3}^{\eta_{+}}+I_{3,3}^{\eta_{-}} . & &
\end{array}
$$

\section{Vertex Adjacent Case}

In this case, we orient the elements so that the singular point is $\eta=-1$ and $\eta^{\prime}=-1$ and we introduce the following separate polar coordinate systems for each element:

$$
\eta^{\prime}=\rho_{q} \cos \left(\theta_{q}\right)-1, \xi^{\prime}=\rho_{q} \sin \left(\theta_{q}\right), \quad \text { and } \quad \eta=\rho_{p} \cos \left(\theta_{p}\right)-1, \xi=\rho_{p} \sin \left(\theta_{p}\right)
$$

The singularity is at the common vertex $\rho_{p}=\rho_{q}=0$, justifying the use of one further polar coordinate transformation,

$$
\rho_{p}=\Lambda \cos (\Psi), \quad \rho_{q}=\Lambda \sin (\Psi)
$$

After the new transformation, the distance function takes the form $R=\Lambda \Gamma\left(\theta_{p}, \theta_{q}, \Psi\right)$. The $\left\{\rho_{p}, \rho_{q}\right\}$ domain is a rectangle and the $\Psi$ integration must be taken in two pieces, yielding, after some algebraic manipulation,

$$
I_{p, q}=\int_{0}^{\pi / 3} d \theta_{p} \int_{0}^{\pi / 3} d \theta_{q}\left[\int_{0}^{\Psi_{1}} \Omega_{p, q}\left(L_{1}\right) d \Psi+\int_{\Psi_{1}}^{\pi / 2} \Omega_{p, q}\left(L_{2}\right) d \Psi\right],
$$

where

$$
\Omega_{p, q}\left(L_{m}\right)=\int_{0}^{L_{m}} \cos (\Psi) \sin (\Psi) \psi_{p}\left(\theta_{p}, \Psi, \Lambda\right) \psi_{q}\left(\theta_{q}, \Psi, \Lambda\right) \frac{e^{-j k \Lambda \Gamma\left(\theta_{p}, \theta_{q}, \Psi\right)}}{\Gamma\left(\theta_{p}, \theta_{q}, \Psi\right)} \Lambda^{2} d \Lambda
$$

are analytically evaluated well-behaved functions.

The highly abbreviated derivation presented above will be discussed in detail at the conference presentation.

\section{Numerical Results}

In this section, we will present representative numerical results for the assessment of the direct evaluation method in tackling the problem of the weakly singular integrals evaluation in Galerkin MPIE formulations. More specifically, in Figure 1(a) the relative error in computing the real part (singular portion) of the weakly singular integral (2) with $k=1$ over the edge adjacent triangles defined by the vertices: $\mathbf{r}_{1}=(1,1,0), \mathbf{r}_{2}=(2,1,0), \mathbf{r}_{3}=(1,2,0), \mathbf{r}_{4}=(1,0,0)$ using the direct evaluation 


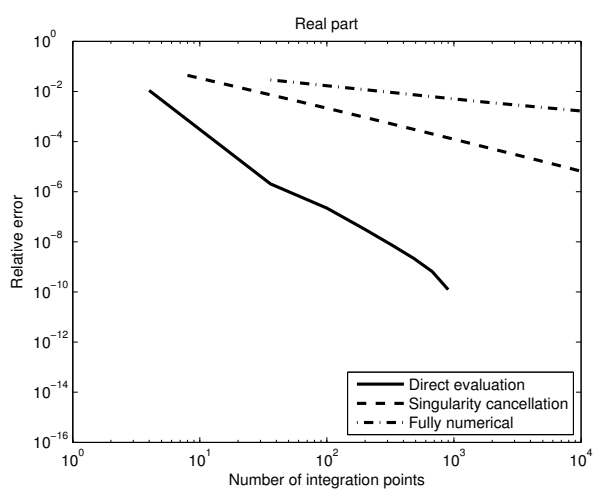

(a) Edge adjacent case.

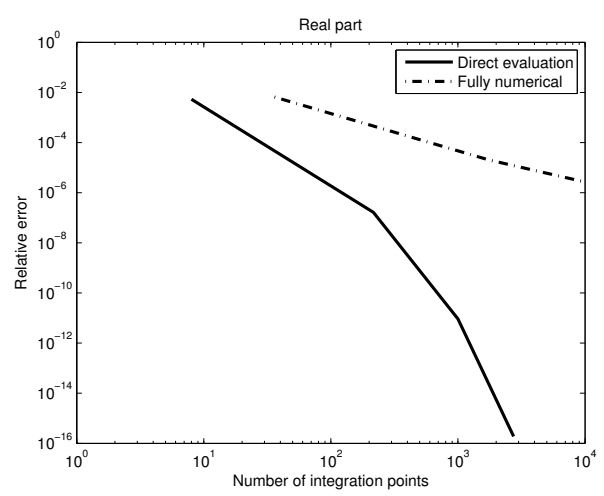

(b) Vertex adjacent case.

Figure 1: Relative error in calculating the real part of $I_{s}^{\text {sing }}$.

method, the singularity cancellation method (here, the direct evaluation method with only one analytical integration) and the fully-numerical method, is presented. In the vertex adjacent case, we proceed to a comparison of the direct evaluation method with the fully-numerical scheme for the computation of the weakly singular integral $I_{s}^{\text {sing }}(k=1)$ over the vertex adjacent triangles defined by the vertices: $\mathbf{r}_{1}=$ $(1,1,1), \mathbf{r}_{2}=(2,1,1), \mathbf{r}_{3}=(1,2,1), \mathbf{r}_{4}=(0,1,1), \mathbf{r}_{5}=(0,2,1)$, as shown in Figure 1(b). It is important to mention that for both examples the converged solution of the direct evaluation scheme is taken as a reference. Again, as expected, the direct evaluation method succeeds in an accurate evaluation together with a greatly reduced computational burden. Therefore, the treatment of the edge adjacent and vertex adjacent integration with the help of the direct evaluation method is fully justified not only for the presentation of a unified approach (for coincident, edge adjacent and vertex adjacent integrations), but also because of the overall superior behavior.

\section{References}

[1] S. M. Rao, D. R. Wilton, and A. W. Glisson, "Electromagnetic scattering by surfaces of arbitrary shape," IEEE Trans. Antennas Propag., vol. 30, no. 5, pp. 409-418, May 1982.

[2] J. R. Mosig, R. C. Hall, and F. E. Gardiol, Numerical analysis of microstrip patch antennas. in Handbook of Microstrip Antennas, James and Hall, Eds. London: IEE-Peter Peregrinus, 1989.

[3] A. G. Polimeridis and T. V. Yioultsis, "On the direct evaluation of weakly singular integrals in Galerkin mixed potential integral equation formulations," IEEE Trans. Antennas Propag., vol. 56, no. 9, pp. 3011-3019, Sep. 2008.

[4] L. J. Gray, A. Salvadori, A. V. Phan, and A. Mantic, "Direct evaluation of hypersingular Galerkin surface integrals. II," Electronic Journal of Boundary Elements, vol. 4, no. 3, pp. 105-130, 2006. 